\title{
ON THE PROGENITORS OF TYPE IA SUPERNOVAE
}

\author{
X.-D. LI \\ Department of Astronomy, Nanjing University \\ Nanjing 210093, P. R. China \\ AND \\ E. P. J. VAN DEN HEUVEL \\ Astronomical Institute, University of Amsterdam \\ 1098 SJ Amsterdam, The Netherlands
}

\section{Introduction}

Supersoft X-ray sources (hereafter SSS) are a class of luminous (bolometric luminosity $\sim 10^{37}-10^{38} \mathrm{erg} \mathrm{s}^{-1}$ ) objects with a characteristic radiation temperature of 30 to $60 \mathrm{eV}$ (Hasinger 1994; Kahabka \& Trümper 1996). The most popular model for SSS is that they are massive white dwarfs steadily burning nuclear fuel accreted from a more massive binary companion at a rate near or above the Eddington limit (van den Heuvel et al. 1992).

Detailed population studies on SSS and their evolutionary connection with type Ia supernovae (SNe Ia) have been made by Rappaport et al. (1994), Yungelson et al. (1996) and Di Stefano \& Nelson (1996). Recently, Hachisu, Kato \& Nomoto (1996) found a new strong wind solution when the mass accretion rate of a white dwarf exceeds a critical value, thus avoiding the common envelope evolution. Motivated by the work of Hachisu et al. (1996), we have performed evolutionary calculations of white dwarf binaries, to investigate the population of the possible SN Ia progenitors.

\section{The model}

We consider a $\mathrm{C}-\mathrm{O}$ white dwarf accreting from a companion star with solar chemical composition ( $\mathrm{X}=0.7, \mathrm{Y}=0.28, \mathrm{Z}=0.02)$. For the outcome of the evolution, we only consider the Chandrasekhar-mass SN Ia explosion of the white dwarf with an initial mass less than $1.2 M_{\odot}$. The companion star is assumed to be lobe-filling, and mass transfer occurs through Roche-lobe overflow. The system can be specified by three parameters: the initial white dwarf mass $M_{\mathrm{WD}, 0}$, the initial donor mass $M_{\mathrm{d}, 0}$ and the orbital period 
$P_{\text {orb }, 0}$ at the beginning of mass transfer. We have followed the evolution of the binary systems for various sets of these parameters, using an updated version of the evolution code developed by Eggleton (1971).

Whether the white dwarf can grow in mass is determined by the "accumulation ratio" $\alpha$, the fraction of the accreted hydrogen that converts into elements heavier than helium. The value of $\alpha$ is related to mass loss from the white dwarf during hydrogen and helium burning, i.e., $\alpha \equiv \alpha_{\mathrm{H}} \alpha_{\mathrm{He}}$, where $\alpha_{\mathrm{H}}$ and $\alpha_{\mathrm{He}}$ are the fraction of the transferred mass accumulated during hydrogen and helium burning, respectively.

It has been shown (e.g. Nomoto et al. 1979) that steady hydrogen burning on the white dwarf surface can occur when $\dot{M}_{\text {low }} \leq \dot{M} \leq \dot{M}_{\text {up }}$, where $\dot{M}$ is the mass transfer rate, $\dot{M}_{\mathrm{up}} \simeq 8.5 \times 10^{-7}\left(M_{\mathrm{WD}} / M_{\odot}-0.52\right) M_{\odot} \mathrm{yr}^{-1}$ and $\dot{M}_{\text {low }} \simeq 0.4 \dot{M}_{\text {up }}$. As $\dot{M}<\dot{M}_{\text {low }}$, hydrogen burning is unstable and occurs in flashes, in which part of the white dwarf envelope may be ejected. For the hydrogen accumulation ratio $\alpha_{\mathrm{H}}$, we have taken the following assumptions: (1) if $\dot{M}>\dot{M}_{\text {up }}$, we adopt the strong wind solution by Hachisu et al. (1996), which allows burning of hydrogen into helium at a rate limited to $\dot{M}_{\text {up }}$, the excess material being blown off in the wind, i.e., $\alpha_{\mathrm{H}}=\dot{M}_{\text {up }} / \dot{M}$; (2) if $\dot{M} \leq \dot{M}_{\text {up }}$, we fit the data of the accumulation ratio calculated by Prialnik \& Kovetz (1995) for hydrogen burning and flashes (for each combination of $M_{\mathrm{WD}}$ and $\dot{M}$ ) with the following conditions: (i) when $\dot{M}$ declines below $3 \times 10^{-8} M_{\odot} \mathrm{yr}^{-1}$, a strong nova explosion is assumed to occur and no mass accumulates on the surface of the white dwarf, that is $\alpha_{\mathrm{H}}=0$; (ii) when $\dot{M}=\dot{M}_{\text {up }}, \alpha_{\mathrm{H}}=1$.

The steady hydrogen burning converts hydrogen into helium on top of the $\mathrm{C}-\mathrm{O}$ white dwarf. When its mass of the helium layer reaches a certain value, helium ignites. Helium shell burning is unstable if $\dot{M}_{\mathrm{He}} \leq 10^{-6} M_{\odot}$ $\mathrm{yr}^{-1}$, and a flash grows, during which part of the envelope mass is blown off (Kato, Saio \& Hachisu 1989). For the helium accumulation ratio $\alpha_{\mathrm{He}}$, we use the results given by Kato et al. (1989).

Generally we have two kinds of products: (1) $M_{\mathrm{WD}}$ grows to $1.4 M_{\odot}$, producing a SN Ia; (2) the calculations are stopped before $M_{\mathrm{WD}}$ reaches $1.4 M_{\odot}$ because $\dot{M}$ exceeds $\sim 10^{-4} M_{\odot} \mathrm{yr}^{-1}$ at which there is no wind solution, or $\dot{M}$ declines below $3 \times 10^{-8} M_{\odot} \mathrm{yr}^{-1}$, at which a strong nova explosion is assumed to occur, or for a low-mass $\left(\leq 1.5 M_{\odot}\right)$ donor, the helium core mass reaches $0.46 M_{\odot}$.

\section{Results and discussion}

An example of the calculated evolution $\left(M_{\mathrm{WD}, 0}=1.0 M_{\odot}, M_{\mathrm{d}, 0}=2.5 M_{\odot}\right.$, $\left.P_{\text {orb }, 0}=1.6 \mathrm{~d}\right)$ is plotted in Fig. 1 . In this case the white dwarf can grow to $1.4 M_{\odot}$ to trigger a SN Ia. Despite of an initial mass ratio of 2.5 , the 


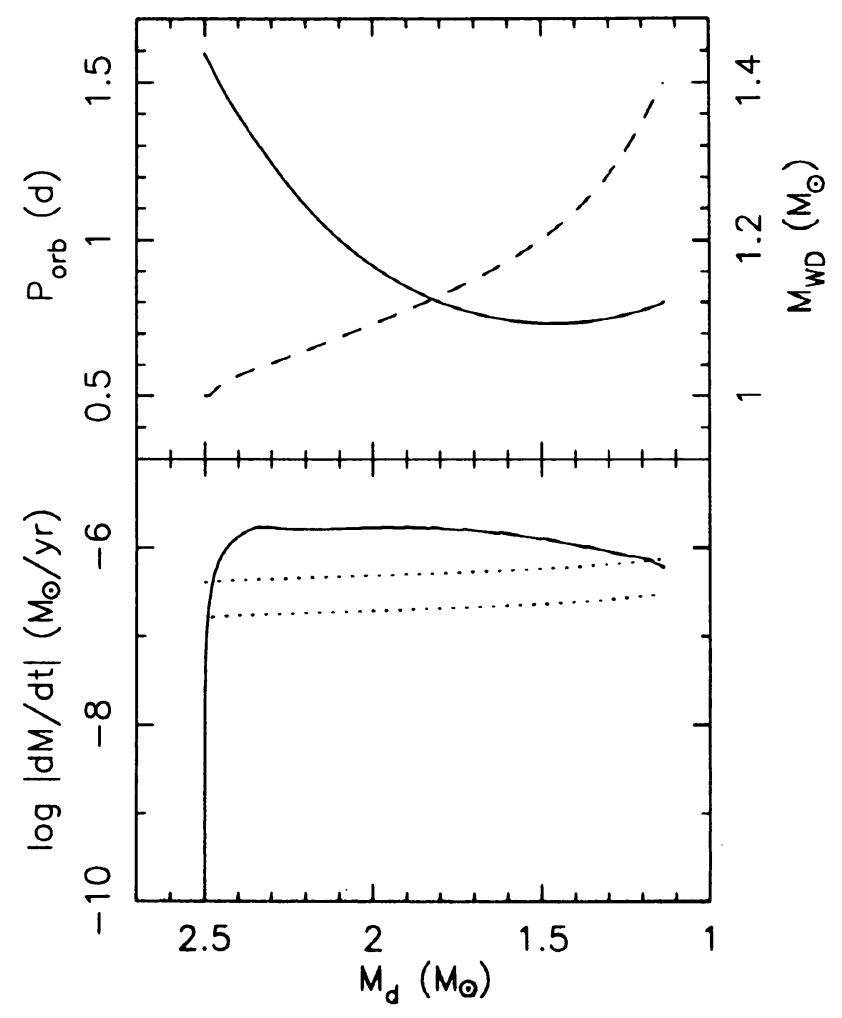

Figure 1. Evolution of the white dwarf binary with $M_{\mathrm{WD}, 0}=1 M_{\odot}, M_{\mathrm{d}, 0}=2.5 M_{\odot}$ and $P_{\text {orb }, 0}=1.6 \mathrm{~d}$. Upper panel: solid and dashed curves denote the evolution of the orbital period and the mass of the white dwarf, respectively. Lower panel: evolution of the mass transfer rate. The dotted lines represent the steady burning region.

mass transfer is stabilized by the strong wind, and maintains a rate around $10^{-6} M_{\odot} \mathrm{yr}^{-1}$ during the whole evolution, i.e., nearly above the steady burning region. A large fraction $(\sim 70 \%)$ of the transferred matter is blown off in the wind. The orbital period decreases to $\sim 0.7 \mathrm{~d}$ and then increases before the white dwarf mass reaches $1.4 M_{\odot}$. A possible candidate that shows a similar evolutionary path is the transient supersoft X-ray source RX J0513.9-6951 in the Large Magellanic Cloud (Alcock et al. 1996; Cowley et al. 1996; Southwell et al. 1996).

Figure 2 presents another example of the evolution for $M_{\mathrm{WD}, 0}=1.2 M_{\odot}$, $M_{\mathrm{d}, 0}=1 M_{\odot}$, and $P_{\text {orb }, 0}=300 \mathrm{~d}$. The mass transfer rate, as in Fig. 1 , is high enough to maintain stable hydrogen burning and weak helium flashes to increase the white dwarf mass. The orbital period increases as mass is transferred from the less massive donor to the white dwarf. Similar features can also be found in Fig. 2 in Hachisu et al. (1996).

We summarize the final outcome of our evolutionary calculations in 


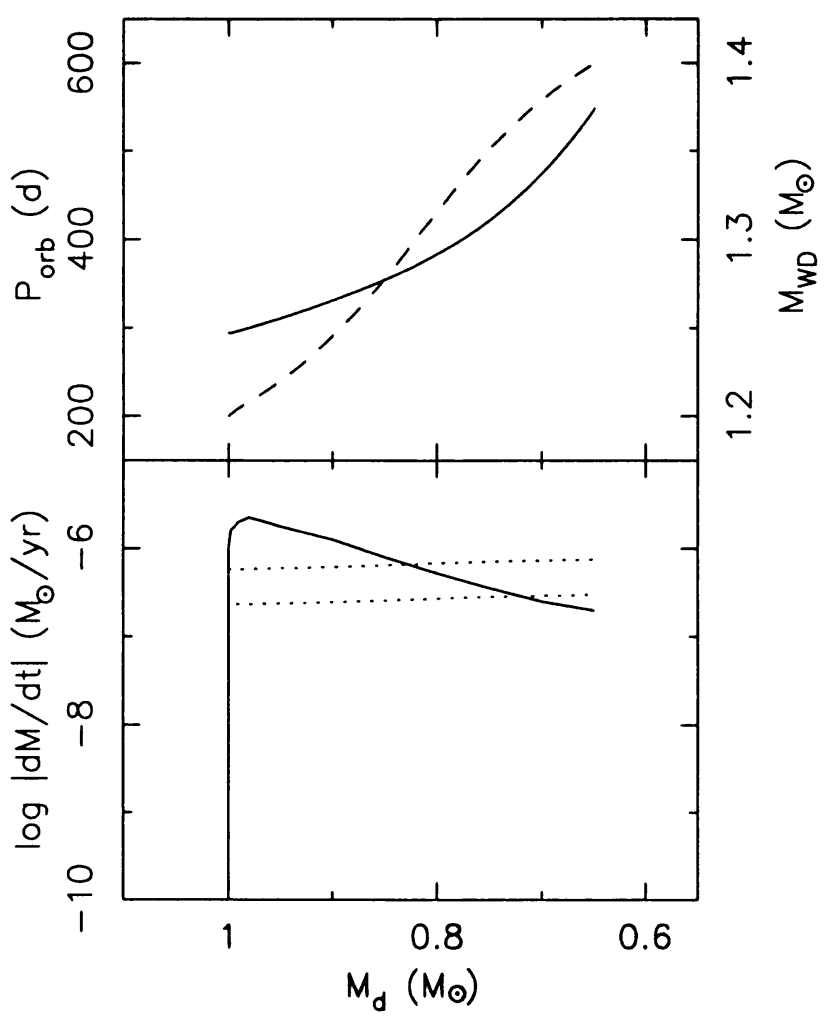

Figure 2. Same as Fig. 1 , but $M_{\mathrm{WD}, 0}=1.2 M_{\odot}, M_{\mathrm{d}, 0}=1.0 M_{\odot}$ and $P_{\mathrm{orb}, 0}=300 \mathrm{~d}$.

Fig. 3, showing the distribution of the progenitor systems of SNe Ia in the $M_{\mathrm{d}, 0}-P_{\text {orb, } 0}$ diagram. It can be seen that there are two "islands", or two types of progenitor systems in the $M_{\mathrm{d}, 0}-P_{\mathrm{orb}, 0}$ diagram. One is close binaries that contain a massive $\left(M_{\mathrm{d}, 0} \sim 2-3.5 M_{\odot}\right)$ donor with an initial orbital period of several tenth of a day to several days, and mass transfer occurring in Case A and Case B (see dotted curves in the figure). The initial white dwarf mass that may increase to $1.4 M_{\odot}$ in this case can actually be as low as $0.9 M_{\odot}$. The other is low-mass $\left(M_{\mathrm{d}, 0} \sim 1 M_{\odot}\right)$ binaries with long orbital period (tens to hundreds of days), in which the donor is a red giant, i.e., in shell hydrogen burning phase, and the required initial mass of the white dwarf is $\sim 1.2 M_{\odot}$, a bit larger than the value $\left(0.9 M_{\odot}\right)$ suggested by Hachisu et al. (1996).

Using the method suggested by Iben \& Tutukov (1984), one can estimate the birth rate of SNe Ia, based on Fig. 3, to be $\sim 2 \times 10^{-3} \mathrm{yr}^{-1}$ in our Galaxy, roughly consistent with the observed rate. The two types of progenitor systems in Fig. 3 can also qualitatively account for SN Ia explosions in both spiral and elliptical galaxies. 


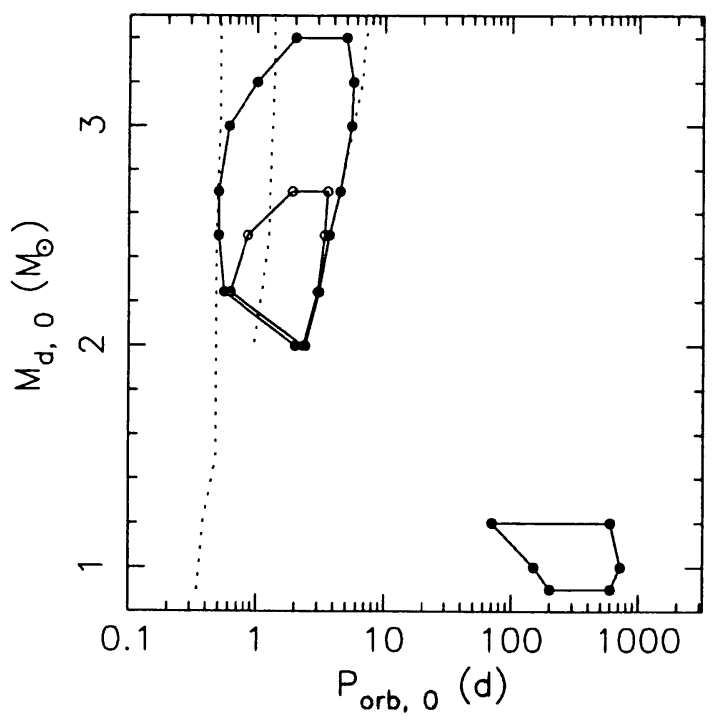

Figure 3. Distribution of the progenitors of SNe Ia in the $M_{\mathrm{d}, 0}-P_{\mathrm{orb}, 0}$ diagram. The filled dots denote the boundary of the initial orbital period at the beginning of the mass transfer, for a white dwarf of $1.2 M_{\odot}$ initial mass with a specific companion star, and circles for $1 M_{\odot}$ white dwarfs. The dotted lines represent the boundary of mass transfer in Case A and Case B (from left to right).

\section{References}

Alcock, C. et al. (1996) MNRAS, 280, L49

Cowley, A. P. et al. (1996) in IAU Symp. 165, Compact Stars in Binaries, eds. J. van Paradijs, E. P. J. van den Heuvel \& E. Kuulkers (Dordrecht: Kluwer), 439

Di Stefano, R. and Nelson, N. A. (1996) in Supersoft X-ray Sources, ed. J. Greiner (Berlin: Springer), 3

Eggleton, P. P. (1971) MNRAS, 151, 351

Hachisu, I., Kato, M. and Nomoto, K. (1996) ApJ, 470, L97

Hasinger, G. 1994, Mod. Rev. Astron., 7, 129

Iben, I. Jr. and Tutukov, A. V. 1984, ApJS, 54, 335

Kahabka, P and Trümper, J. (1996) in IAU Symp. 165, Compact Stars in Binaries, eds. J. van Paradijs, E. P. J. van den Heuvel \& E. Kuulkers (Dordrecht: Kluwer), 425

Kato, M., Saio, H. and Hachisu, I. (1989) $A p J, 340,509$

Nomoto, K., Nariai, K. and Sugimoto, D. (1979) PASJ, 31, 287

Prialnik, D. and Kovetz, A. 1995, $A p J, 445,789$

Rappaport, S. A., Di Stefano, R. and Smith, M. (1994) ApJ, 426, 692

Southwell, K. A. et al. (1996) ApJ, 470, 1065 
van den Heuvel, E. P. J. et al. (1992) $A \& A, 262,97$

Yungelson, L. et al. (1996) $A p J$, 466, 890 